\title{
General Drift-Diffusion Theory of the Current Density in Schottky Diodes
}

\author{
Piet Van Mieghem
}

\begin{abstract}
A new general expression for the current in Schottky diodes due to drift-diffusion but neglecting tunneling is presented. A precise expression for the ideality factor is derived. The temperature dependence of important quantities in both the degenerate (heavy doping) and nondegenerate (Boltzmann) limit is examined. In the degenerate regime, the temperature independence of the saturation current is shown to complicate the extraction of the barrier height from the current-voltage characteristics.
\end{abstract}

\section{INTRODUCTION}

A LTHOUGH the theory of Schottky diodes (or more generally metal-semiconductor interfaces) has a long and rich history [1], [2], a complete understanding is still not achieved. Most of the literature concentrates on the determination of the barrier height between the metal and semiconductor interface, but relatively little effort has been devoted to heavily doped diodes. Very often, weak temperature dependence of the current has been attributed to tunneling although this is not true in general as will be demonstrated here.

So far a Schottky diode theory using the Fermi-Dirac statistics everywhere has not appeared yet. Transport equations for heavily doped semiconductors have first been derived by Van Overstraeten et al. [3] to include heavy doping effects such as bandgap narrowing [8] and bandtailing [9]. The earlier evolution in the theory of transport in heavily doped semiconductors as reviewed by Mertens et al. [4] is based upon the drift-diffusion theory and characterized by an attempt to include degeneracy and heavy doping effects in the framework of Boltzmann statistics through the definition of various effective quantities in order to maintain the simple Boltzmann expressions. The results were mainly used in the study of bipolar transistors.

In this paper, we demonstrate that a new general and exact expression for the electron current density in a Schottky diode can be derived from the diffusion-drift current equation, however neglecting tunneling. The theory assumes a homogenous metal-semiconductor interface. We show that, as a consequence of the Fermi-Dirac statistics, the ideality factor $m$ varies as $m \sim T^{-\beta}$ where $\beta$ is a doping concentration dependent function ranging from $\beta=0$ for very low doping

\footnotetext{
Manuscript received February 25, 1994; revised July 1, 1994. The review of this paper was arranged by Associate Editor J. R. Hauser. This work was supported in part by NATO, the Belgian National Fund for Scientific Research (NFWO) and in part by the Interuniversity Micro Electronics Center (IMEC) in Belgium.

The author is with Alcatel Bell, B-2018 Antwerpen, Belgium. This work was done at the Massachusetts Institute of Technology, Cambridge, MA 02139 USA

IEEE Log Number 9405955.
}

concentrations where Boltzmann statistics are valid to $\beta=1$ for heavily doped diodes. Previously an inverse $T$-law for the ideality factor was ascribed to tunneling [2]. In addition, our analysis emphasizes that the temperature independence of the current for high doping concentrations complicates the extraction of the barrier height from current-voltage characteristics significantly. As a conclusion, we point out that the knowledge of the barrier height alone is insufficient except both the doping concentration and the temperature interval from which the data is extracted are specified.

These new insights may help to improve the current understanding for heavily doped Schottky diodes.

\section{INTEGRAL FORM OF THE DRIFT-DIFFUSION EQUATION}

When applying a voltage $V$ across a n-type semiconductor, the electric current density due to electrons in one-dimension, $J_{n}(V)$, satisfies the drift-diffusion equation [5]

$$
J_{n}(V)=q \mu_{n}(x) n(x ; V) \mathcal{E}(x ; V)+q D_{n}(x) \frac{d n(x ; V)}{d x}
$$

where $\mu_{n}(x)$ is the electron mobility, $D_{n}(x)$ is the electron diffusion coefficient, $n(x ; V)$ denotes the density of electrons as a function of distance $x$ and of applied voltage $V$ and $\mathcal{E}(x ; V)$ is the electric field at position $x$ corresponding to the applied voltage $V$. For the sake of brevity, we limit the discussion to n-type semiconductors. The adoption of the one-dimensional drift-diffusion equation (1) is the sole approximation made. The voltage independent mobility excludes cases where velocity saturation occurs. But, more important, the drift-diffusion equation (1) does not account for tunneling that undoubtedly plays a role in heavily doped Schottky diodes at low temperatures.

At a constant temperature $T$ in equilibrium $(V=0)$, there is no current or $J_{n}(0)=0$. This condition relates the electron mobility and diffusion coefficient as

$$
\frac{\mu_{n}(x)}{D_{n}(x)}=\frac{\partial \ln [n(x ; 0)]}{\partial \psi(x ; 0)} \equiv f(x)
$$

where the electrostatic potential $\psi(x ; V)$ and the electric field are linked by $\mathcal{E}(x ; V)=-\frac{d \psi(x ; V)}{d x}$. When assuming Boltzmann statistics, (2) reduces to the well known Einstein relation [14] $f(x)=f=\frac{q}{k_{B} T}$. With Fermi-Dirac statistics 
and a parabolic density of states ${ }^{1} f(x)$ can be rewritten as

$$
f(x)=\frac{q}{k_{B} T} \frac{F_{-1 / 2}\left[\frac{q\left[\psi(x ; 0)-\phi_{n}(x ; 0)\right]}{k_{B} T}\right]}{F_{1 / 2}\left[\frac{q\left[\psi(x ; 0)-\phi_{n}(x ; 0)\right]}{k_{B} T}\right]}
$$

where $\phi_{n}(x ; V)$ is the quasi-fermi level for electrons at position $x$ when the voltage $V$ is applied over the diode and $\phi_{n}(x ; 0)=-E_{F} / q$ for a Fermi level $E_{F}$. Since $\frac{F_{p}(y)}{F_{q}(y)} \leq 1$ for real $q>p>-1[17]$ we find the upper bound $f(x) \leq \frac{q}{k_{B} T}$. In addition $f(x)$ is positive. Thus

$$
0<f(x) \leq \frac{q}{k_{B} T}
$$

This implies that the Boltzmann limit gives the maximal value for $f(x)$.

As demonstrated in the appendix, the electron current density $J_{n}(V)$ in (1) can be rewritten as

$$
J_{n}(V)=J_{s}(V)\left(\frac{n(b ; V)}{n(a ; V)} \exp \left[\int_{a}^{b} \mathcal{E}(x ; V) f(x) d x\right]-1\right)
$$

with

$$
J_{s}(V)=\frac{q n(a ; V)}{\int_{a}^{b} \frac{d x}{D_{n}(x)} \exp \left[\int_{a}^{x} \mathcal{E}(u ; V) f(u) d u\right]}
$$

where $a$ and $b$ are arbitrary points in the semiconductor. Relation (5) being mathematically equivalent to (1), can include besides the Fermi-Dirac statistics all heavy doping effects as many body interactions [8] and bandtails [9]. One may wonder if (5) can be expanded around the low density limit (Boltzmann statistics) so that the degenerate case may be treated as a perturbation. Since we can show (see appendix) that the number of electrons can be rewritten in terms of Fermi-Dirac integrals for an arbitrary density of states and since the Fermi-Dirac integrals $F_{p}(y)$ do not have a Dirichlet series for $y>0$ as shown by Dingle [10], we conclude that the degenerate regime is both mathematically and physically disconnected from the nondegenerate regime.

For Boltzmann statistics where $n(x ; V)=N_{c} \exp$ $\left[\frac{q\left[\psi(x ; V)-\phi_{n}(x ; V)\right]}{k_{B} T}\right]$ and $f(x)=\frac{\mu_{n}(x)}{D_{n}(x)}=\frac{q}{k_{B} T}$ equation

\footnotetext{
${ }^{1}$ A more general treatment that includes an arbitrary density of states is possible by introducing our -transform (see appendix and [6], [7]).
}

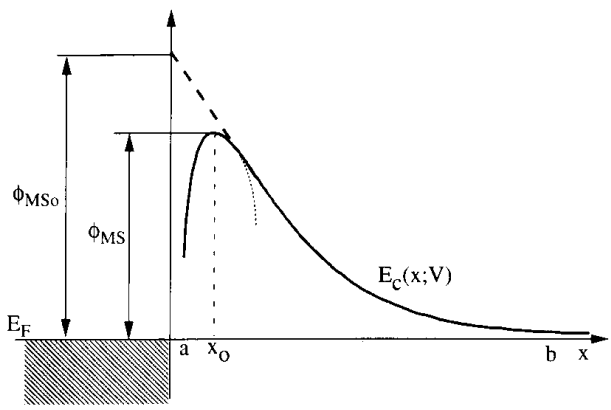

Fig. 1. Conduction band energy $E_{c}(x: V)$ versus distance $x$. The dotted line shows the profile neglecting the Schottky effect $\left(x_{0}=0\right)$.

(5) reduces to

$$
J_{n B}(V)=J_{s B}(V)\left(\exp \left[\frac{q\left[\phi_{n}(a ; V)-\phi_{n}(b ; V)\right]}{k_{B} T}\right]-1\right)
$$

with

$$
J_{s B}(V)=\frac{q N_{c} \exp \left[-\frac{q \phi_{n}(a ; V)}{k_{B} T}\right]}{\int_{a}^{b} \frac{d x}{D_{n,}(x)} \exp \left[-\frac{q \psi(x ; V)}{k_{B} T}\right]}
$$

\section{CuRrent IN AN N-TyPE SCHOTtKy DiOdE}

We first consider Boltzmann statistics in order to compare our results with the expression of Crowell and Sze [11] or Taylor and Simmons [12]. Then, we will generalize the Boltzmann results using (6).

A realistic sketch of the conduction band profile is drawn in Fig. 1 (see also Fig. 6 on pp. 253 in [14] and potential profiles computed in [20]). Let us choose $a<x_{0}$ and $b$ in the quasi-neutral region. The point $x_{0}$ is the position in the semiconductor close to the metal interface where the potential $\psi(x)=-E_{c}(x) / q$ reaches its minimum (see Fig. 1). Initially, we rewrite and physically interpret (8) relying on the shape of the sketch in Fig. 1. Afterwards, we will discuss the influence of the precise location of $a$ and $b$ and of the interface boundary conditions.

Equation (8) can be rewritten as (see (9) at bottom of page). The first integral in the denominator $I_{1}=$ $\exp \left(\frac{q \psi\left(x_{0} ; V\right)}{k_{B} T}\right) \int_{a}^{x_{0}} \frac{d x}{D_{n}(x)} \exp \left(-\frac{q \psi(x ; V)}{k_{B} T}\right)$ can be approximated as follows. Around its minimum $x_{0}$ the potential increases rapidly. This observation suggests to use the method of the steepest descent (4.6 in [15]). We expand the potential

$$
\begin{aligned}
J_{s B}(V) & =\frac{q N_{c} \exp \left(\frac{q\left[\psi(a ; V)-\phi_{n}(a ; V)\right]}{k_{B} T}\right)}{\exp \left(\frac{q\left[\psi(a ; V)-\psi\left(x_{0} ; V\right)\right]}{k_{B} T}\right) \exp \left(\frac{q \psi\left(x_{0} ; V\right)}{k_{B} T}\right) \int_{a}^{b} \frac{d x}{D_{n}(x)} \exp \left(-\frac{q \psi(x ; V)}{k_{B} T}\right)} \\
& =\frac{q N_{c} \exp \left(\frac{q\left[\psi(a ; V)-\phi_{n}(a ; V)\right]}{k_{B} T}\right) \exp \left(-\frac{q\left[\psi(a ; V)-\psi\left(x_{0} ; V\right)\right]}{k_{B} T}\right)}{\exp \left(\frac{q \psi\left(x_{0} ; V\right)}{k_{B} T}\right)\left[\int_{a}^{x_{0}} \frac{d x}{D_{n}(\boldsymbol{x})} \exp \left(-\frac{q \psi(x ; V)}{k_{B} T}\right)+\int_{x_{0}}^{b} \frac{d x}{D_{n}(x)} \exp \left(-\frac{q \psi(x ; V)}{k_{B} T}\right)\right]} .
\end{aligned}
$$


$\psi(x)$ around the minimum $x_{0}$ where $\psi^{\prime \prime}(x)>0$ and obtain

$$
\begin{aligned}
I_{1} & \approx \int_{u}^{x_{0}} \frac{d x}{D_{n}(x)} \exp \left(-\frac{q \psi^{\prime \prime}\left(x_{0} ; V\right)}{2 k_{B} T}\left(x-x_{0}\right)^{2}\right) \\
& \approx \frac{1}{2} \int_{a}^{2 x_{0}-a} \frac{d x}{D_{n}(x)} \exp \left(-\frac{q \psi^{\prime \prime}\left(x_{0} ; V\right)}{2 k_{B} T}\left(x-x_{0}\right)^{2}\right) \\
& \approx \frac{1}{2 D_{n}\left(x_{0}\right)} \int_{-\infty}^{\infty} d x \exp \left(-\frac{q \psi^{\prime \prime}\left(x_{0} ; V\right)}{2 k_{B} T}\left(x-x_{0}\right)^{2}\right) \\
& =\frac{1}{2 D_{n}\left(x_{0}\right)} \sqrt{\frac{2 \pi k_{B} T}{q \psi^{\prime \prime}\left(x_{0} ; V\right)}} .
\end{aligned}
$$

We use the Poisson equation $\psi^{\prime \prime}(x)=-\frac{q}{\epsilon} n_{\text {net }}(x)$ where $q n_{\text {net }}$ denotes the total net charge. Since $\psi^{\prime \prime \prime}\left(x_{0}\right)>0$ we have that $n_{\text {net }}<0$ regardless of the doping concentration of the semiconductor. The charge distribution where the curvature of the potential is positive consists mainly of electrons. The underlying physical phenomena, image charge and correlation effects, have been analyzed in detail by Vinter [13]. In this seemingly metallic region we can define a "Debye length $L_{D m}$ " as

$$
L_{D m}=\sqrt{\frac{\epsilon_{s} k_{B} T}{q^{2} n_{\text {net }}}}
$$

and obtain for $I_{1}$

$$
I_{1} \approx \sqrt{\frac{\pi}{2}} \frac{L_{D m}}{D_{n}\left(x_{0}\right)}
$$

The diffusion coefficient can be written as $D_{n}\left(x_{0}\right)=l_{n} v_{\text {th }}$ where $l_{n}$ and $v_{\mathrm{th}}=\sqrt{\frac{k_{B} T}{2 \pi m^{*}}}$ denotes the electron mean free path and the thermal velocity in a material with effective mass $m^{*}$, respectively. Thus, we have

$$
I_{1} \approx \sqrt{\frac{\pi}{2}} v_{\mathrm{th}}^{-1} \frac{L_{D m}}{l_{n}} \equiv v_{m}^{-1}
$$

where $v_{m}$ describes the velocity of electrons at $x_{0}$.

The second integral, $I_{2}=\exp \left(\frac{q \psi\left(x_{0} ; V\right)}{k_{B} T}\right) \int_{x_{0}}^{b} \frac{d x}{D_{n}(x)}$ $\exp \left(-\frac{q \psi(r ; \mathrm{V})}{k_{B} T}\right) \equiv v_{e}^{-1}$, has been thoroughly investigated in the literature [1] and approximated as

$$
v_{\epsilon} \approx 2 v_{\mathrm{th}} \frac{l_{n}}{L_{D}} \sqrt{\frac{q\left[V_{\mathrm{bi}}-V\right]}{k_{B} T}-1}
$$

where $L_{D}$ denotes the usual Debye length [14] and $V_{\mathrm{bi}}$ is the built-in potential. The quantity $v_{e}$ describes the velocity of electrons in the depletion layer due to a drift-diffusion mechanism. Since both defined velocities are weakly voltage dependent, it is in general difficult to determine which is dominant. The two extreme regimes are broadly discussed in [1]. The approximations for $I_{1}$ and $I_{2}$ simplify the expression of the saturation current considerably,

$$
\begin{aligned}
J_{s B}(V)= & \frac{q N_{c}}{v_{m}^{-1}+v_{\epsilon}^{-1}} \exp \left(\frac{q\left[\psi(a ; V)-\phi_{n}(a ; V)\right]}{k_{B} T}\right) \\
& \times \exp \left(-\frac{q\left[\psi(a ; V)-\psi\left(x_{0} ; V\right)\right]}{k_{B} T}\right) .
\end{aligned}
$$

At this point we introduce the concept of a barrier height $\Phi_{\text {MS }}$ between the metal and semiconductor. From Fig. 1, we read that $\Phi_{\mathrm{MS}}=q\left[\psi(a ; V)-\psi\left(x_{0} ; V\right)\right]$. So far the precise location of the point $a$ was not required. Since the region $\left[0, x_{0}\right]$ is shown to be effectively metallic, the potential $\psi(a ; V)$ can be choosen to coincide with the quasi-fermi level in the metal that acts as potential reference $\left(\phi_{n}(a ; V)=0\right)$. Hence, we choose the point $a$ sufficiently close to the metallurgical junction. Our final result which was orginally due to Crowell and Sze [11] except with $v_{m}$ replaced.by $v_{\mathrm{th}}$, is

$$
J_{n}=\frac{q N_{c}}{v_{m}^{-1}+v_{e}^{-1}} \exp \left(-\frac{\Phi_{\mathrm{MS}}}{k_{B} T}\right)\left[\exp \left(\frac{q V}{k_{B} T}\right)-1\right] .
$$

It is instructive to briefly deduce the Simmons and Taylor formula. They essentially make two assumptions. First they neglect the Schottky effect [14] which means that $x_{0}$ lies precisely at the metallurgical junction and that the potential only exhibits a negative curvature (see dotted curve in Fig. 1). The omission of the Schottky effect causes only a small error as shown by Crowell and Sze [11]. Secondly, the metal interface current is approximated as

$$
J=q v_{\mathrm{th}}[n(a ; V)-n(a ; 0)] .
$$

Substituting the expression for the interface density of electrons $n(a ; V)$ from (5) into (17) and solving for $J_{n}=J$ yields

$$
J_{n}=\frac{q n(a ; 0)}{v_{\mathrm{th}}^{-1}+v_{e}^{-1}}\left[\frac{n(b ; V)}{n(a ; 0)} \exp \left(\int_{a}^{b} \mathcal{E}(x ; V) f(x) d x\right)-1\right]
$$

The Boltzmann approximation of (18) gives the Crowell-Sze or Simmons-Taylor formula

$$
J_{n}=\frac{q N_{c}}{v_{\mathrm{th}}^{-1}+v_{\mathrm{e}}^{-1}} \exp \left(-\frac{\Phi_{\mathrm{MS}}}{k_{B} T}\right)\left[\exp \left(\frac{q V}{k_{B} T}\right)-1\right] .
$$

$$
\begin{aligned}
J_{s}(V) & =\frac{q n(a ; V) \exp \left[-\int_{a}^{x_{0}} \mathcal{E}(u ; V) f(u) d u\right]}{\int_{a}^{x_{0}} \frac{d x}{D_{n}(x)} \exp \left[-\int_{x}^{x_{0}} \mathcal{E}(u ; V) f(u) d u\right]+\int_{x_{0}}^{b} \frac{d x}{D_{n}(x)} \exp \left[\int_{x_{0}}^{x} \mathcal{E}(u ; V) f(u) d u\right]} \\
& \equiv \frac{q n(a ; V)}{v_{m}^{-1}+v_{e}^{-1}} \exp \left[-\int_{a}^{x_{0}} \mathcal{E}(u ; V) f(u) d u\right]
\end{aligned}
$$




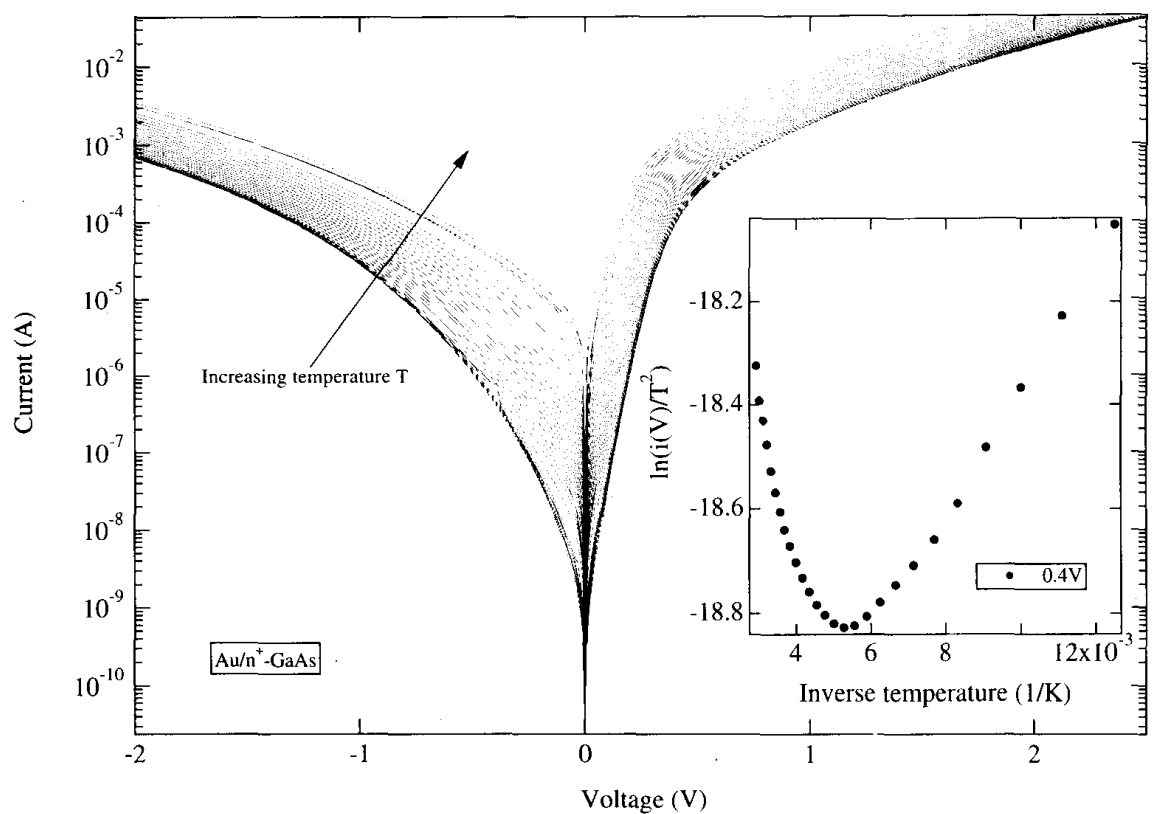

Fig. 2. The current-voltage characteristic from $80 \mathrm{~K}$ to $340 \mathrm{~K}$. The insert $\ln \left[i(v) / T^{2}\right]$ versus $1 / T$ exhibits clearly two different temperature regimes: thermionic transport at high temperature and a degenerate temperature independent behavior at low temperature. The latter temperature regime also includes tunneling.

This equation implies that the reference for the quasi-fermi level is the Fermi level in the metal $\left(\phi_{n}(a ; 0)=0\right)$ and that the energy barrier between a metal and semiconductor equals $\Phi_{\mathrm{MS}}=q\left[\psi(a ; 0)-\phi_{n}(a ; 0)\right]$. This result (19) only differs from (16) in the denominator. The generalized Simmons-Taylor result (18) is not an exact integral of the drift-diffusion equation (1) in contrast to (5) with the boundary values explained above. The reason for the discrepancy is that they allow (17) to modify the result.

When examining the general relation (5), the saturation current can be rewritten analogously as (see (20) at bottom of previous page) where we have associated the integrals in the denominator with reciprocal velocities found in the Boltzmann nondegenerate case above. We observe that the barrier height $\Phi_{\mathrm{MS}}$ does not appear explicitly anymore but that the information is concealed in

$$
I_{3}=\int_{a}^{x_{0}} \mathcal{E}(u ; V) f(u) d u
$$

This dimensionless integral describes the work done when crossing the interface potential barrier, however, modulated by the environment through $f(x)$. The consequence for degenerate Schottky diodes is discussed below.

\section{The IdEALITy Factor $m(V, T)$}

A commonly used quantity as figure of merit for the Schottky diode is the ideality factor $m(V, T)$ defined as

$$
m(V, T)=\left[\left(\frac{k_{B} T}{q V}\right) \ln \left|\frac{J_{n}(V)}{J_{s}(V)}+1\right|\right]^{-1}
$$

or with (5)

$$
\frac{1}{m(V, T)}=\frac{k_{B} T}{q V}\left[\ln \left|\frac{n(b ; V)}{n(a ; V)}\right|+\int_{a}^{b} \mathcal{E}(x ; V) f(x) d x\right] .
$$

The definition (22) is often approximated [14] when Boltzmann statistics apply as

$$
m(V, T)=\left[\frac{k_{B} T}{q} \frac{\partial \ln \left[J_{n}(V)\right]}{\partial V}\right]^{-1} \text { for } \quad V>\frac{k_{B} T}{q} .
$$

Another expression follows from (1). After dividing (1) by $q D_{n}(x) n(x ; V)$ and integrating over $[\mathrm{a}, \mathrm{b}]$ we obtain using (23)

$$
\begin{aligned}
J_{n}(V) \int_{a}^{b} \frac{d x}{q D_{n}(x) n(x ; V)}= & \ln \left|\frac{n(b ; V)}{n(a ; V)}\right| \\
& +\int_{a}^{b} \mathcal{E}(x ; V) f(x) d x \\
\equiv & \frac{q V}{m(V, T) k_{B} T} .
\end{aligned}
$$

Defining a voltage dependent 'area' resistance $\rho_{A}(V)=$ $V / J_{n}(V)$ in $\Omega \mathrm{cm}^{2}$, we find from (25) that

$$
\rho_{A}(V)=\frac{m(V, T) k_{B} T}{q} \int_{a}^{b} \frac{d x}{q D_{n}(x) n(x ; V)} .
$$

The current density $J_{n}(V)$ can also be expressed in terms of the quasi-Fermi levels as $J_{n}(V)=-q \mu_{n}(x) n(x ; V) \frac{d \phi_{n}(x)}{d x}$. Performing an analogous operation as explained above yields

$$
J_{n}(V) \int_{a}^{b} \frac{d x}{q \mu_{n}(x) n(x ; V)}=\phi_{n}(a)-\phi_{n}(b) \equiv V
$$


or

$$
\rho_{A}(V)=\int_{a}^{b} \frac{d x}{q \mu_{n}(x) n(x ; V)} .
$$

From (26) and (28) we find an alternative expression for the ideality factor

$$
m(V, T)=\frac{q}{k_{B} T} \frac{\int_{a}^{b} \frac{d x}{q \mu_{n}(x) n(x ; V)}}{\int_{a}^{b} \frac{d x}{q D_{n}(x) n(x ; V)}}=\frac{q}{k_{B} T} \frac{\int_{a}^{b} \frac{d x}{q \mu_{n}(x) n(x ; V)}}{\int_{a}^{b} \frac{f(x) d x}{q \mu_{n}(x) n(x ; \bar{V})}}
$$

Introducing the lower and upper bound (4) for $f(x)$ we obtain $1 \leq m(V, T)<\infty$. For lowly doped good quality Schottky diodes $m(V, T) \rightarrow 1$ as follows from (29) since $f(x) \rightarrow \frac{q}{k_{B} T}$. Hence, when Boltzmann statistics apply, the ideality factor $m(V, T)$ is both temperature and voltage independent.

For heavily doped Schottky diodes, the ideality factor is less meaningful to evaluate the quality of the diode since the rectifying property of the diode decreases rapidly to become an ohmic contact $(m(V, T) \rightarrow \infty)$. Recalling the definition (2) of $f(x)$, the temperature dependence of $m(V, T)$ is related to that of the free carrier concentration $n(x ; V)$. In the degenerate limit $n(x ; V)=N_{c} F_{1 / 2}\left[\frac{q\left[\psi(x ; V)-\phi_{n}(x ; V)\right]}{k_{B} T}\right]$ is almost temperature independent-if we neglect the small temperature dependence of the potential, quasifermi level and mobility-because $N_{c} \sim T^{3 / 2}$ while $F_{1 / 2}\left[\frac{q\left[\psi(x ; V)-\phi_{n}(x ; V)\right]}{k_{B} T}\right] \sim T^{-3 / 2}$ since $F_{p}(y) \sim \frac{y^{p+1}}{\Gamma(p+2)}$ as $y \gg 0$ [17]. From (29) it follows that the ideality factor $m(V, T)$ is inverse proportional with temperature $T$. In the appendix we estimate the ideality factor using the definition (22) rather than (29) because no expansions of $\ln F_{p}(y)$ or $\frac{F_{p-1}(y)}{F_{p}(y)}$ for all $y$ are known. Since for all temperature $T$ of interest a continous transition between both limit cases is possible (by varying the doping concentration) it is quite conceivable that $m(V, T) \sim T^{-\beta}$ where $\beta$ is a certain function of doping concentration obeying $0 \leq \beta \leq 1$.

This temperature dependence of $m(V, T)$ is a universal property of the Fermi-Dirac statistics and should not be confused with the $T_{0}$ anomaly [1] that satisfies $m(V, T)=$ $1+\frac{T_{0}}{T}$ where $T_{0}$ is of the order of $10-50 \mathrm{~K}$. The mechanisms responsible for the $T_{0}$ anomaly are not well understood, although Tung [18], [20] believes that the effect is due to insufficient control of the metal-semiconductor interface causing interfacial inhomogeneities. He further points out that the $T_{0}$ anomaly often occurs in situations dominated by the so-called Fermi-level pinning.

\section{THE BARrier HeIGHT $\Phi_{\text {MS IN }}$ HEAVILY DOPED SCHOTTKY DIODES}

From the previous discussion and the definition of the ideality factor (22) we find that $\frac{J_{n}(V)}{J_{s}(V)}$ is temperature independent in the degenerate limit. Further, invoking the definition of the voltage dependent resistivity and the temperature dependence of the free carriers, it follows that $J_{n}(V)$ is almost temperature independent and, hence, also $J_{s}(V)$. A temperature independent current is generally attributed to tunneling only. Our analysis shows that this statement should be revised.
Let us examine

$$
-k_{B} \ln \left|\frac{J_{n}(V)}{T^{2}}\right|=-k_{B} \ln \left|\frac{J_{s}(V)}{T^{2}}\right|-\frac{q V}{T m(V, T)}
$$

and define an effective barrier height related to a certain temperature range as

$$
\Phi_{\mathrm{eff}}(V, T)=-k_{B} \frac{d}{d T^{-1}} \ln \left|\frac{J_{n}(V)}{T^{2}}\right| .
$$

When Boltzmann statistics apply and $J_{s}(V)=A T^{2} \exp$ $\left(-\frac{\Phi_{\mathrm{MS}}}{k_{B} T}\right)[14]$, we have for voltages larger than $\frac{k_{B} T}{q}$

$$
\Phi_{\mathrm{eff}}(V, T)=\Phi_{\mathrm{MS}}-q V / m
$$

such that the barrier height $\Phi_{M S}$ follows from the extrapolation towards zero voltage.

The temperature independence of degenerate Schottky diodes complicates the extraction of the barrier height $\Phi_{\mathrm{MS}}$ seriously because the both currents $J_{n}(V)$ and $J_{s}(V)$ as well as $I_{3}$ (21) become temperature independent. The standard nondegenerate approach that plots $-k_{B} \ln \left|J_{s}(V) / T^{2}\right|$ versus inverse temperature and then identifies the slope as $\Phi_{M S}$ clearly does not apply anymore.

However, the temperature $T$ can still be used as a tool to extract a high temperature barrier height, because for sufficiently high temperatures a semiconductor becomes again nondegenerate. When plotting $-k_{B} \ln \left|J_{n}(V) / T^{2}\right|$ versus $1 / T$ a clear separation between the temperature independent regime and the thermionic regime can be distinguished as shown in the insert of Fig. 2 for a Au/GaAs Schottky diode with Si donor concentration about $10^{18} \mathrm{~cm}^{-3}$. The Au/GaAs Schottky diode was fabricated without special surface treatment as follows. A $0.4 \mu \mathrm{m}$ thick heavily doped $\mathrm{n}(\mathrm{Si})$-type $\mathrm{GaAs}$ layer was grown by MBE on a commercial $n^{+}-$GaAs substrate. The GaAs surface was prepared by a standard cleaning first with trichloroethylene, then with acetone and with isopropyl alcohol and finally followed by a dehydration bake at $200^{\circ} \mathrm{C}$ for 20 min. under nitrogen flow. A rectangular pattern for the contacts was obtained after a lithography (resist 820-20, developer 9341:1 and deionized water rinsing) by e-beam evaporation of first $20 \mathrm{~nm}$ of $\mathrm{Ti}$ followed by $180 \mathrm{~nm}$ of Au. Finally we have removed the photoresist and have etched $0.3 \mu \mathrm{m}$ of the GaAs surface with $\mathrm{H}_{3} \mathrm{PO}_{4}: \mathrm{H}_{2} \mathrm{O}_{2}: \mathrm{H}_{2} \mathrm{O}$ (3:1:50) to define the junction area. The contacts are on top of the metal and on the back side of the $\mathrm{n}^{+}-\mathrm{GaAs}$ substrate. The currentvoltage characteristic are shown in Fig. 2. The effective barrier height $\Phi_{\text {eff }}(V, T)$ has been fitted from the thermionic regime (high temperatures) by a straight line $-k_{B} \ln \left|J_{n}(V) / T^{2}\right|=$ $A / T+B$. Both the slope $A$ and the constant $B$ are shown in Fig. 3. The low values of $B$ justify the use of (32). The barrier height $\Phi_{\mathrm{MS}}=0.3 \mathrm{eV}$ is found by extrapolation towards zero voltage. The slope of the fit gives $1 / m=0.9$ or an ideality factor of $m=1.1$. 


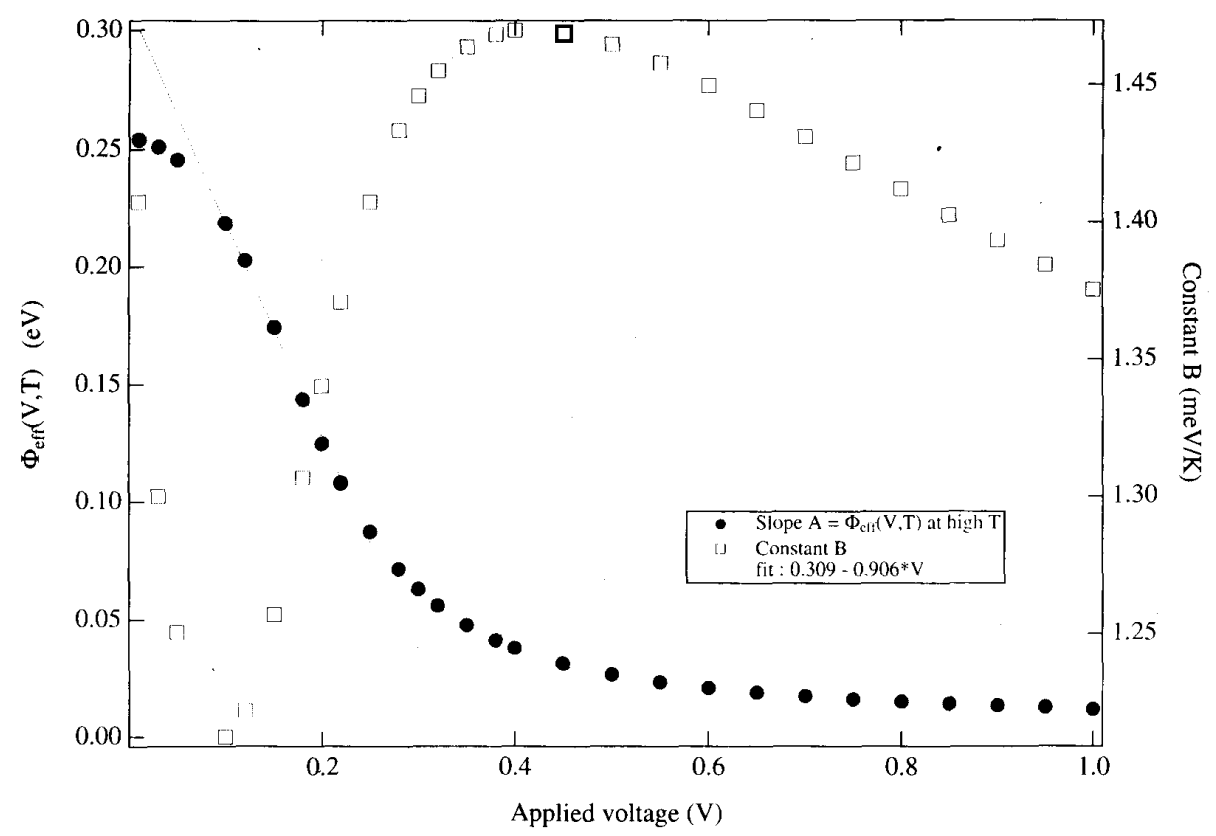

Fig. 3. The effective barrier height $\Phi_{\text {eff }}(V, T)$ versus applied voltage for a Au/n ${ }^{+}-$GaAs Schottky diode extracted from the high-temperature regime by fitting a straight line $A / T+B$. Extrapolation towards zero voltage gives a barrier height of $0.3 \mathrm{eV}$ and the slope indicates that the high temperature ideality factor is about $m=1.1$.

But as generally believed in literature [21], the $\mathrm{Au} / \mathrm{GaAs}$ barrier height is around $0.7 \mathrm{eV}$ (Fermi-level pinning due to the high amount of surface states). Clearly the extracted barrier height is both related to the doping concentration and temperature range where the information is obtained from. Moreover, the ideality factor is found to vary as $m(V, T) \sim T^{-0.8}$ when determined from the current-voltage characteristics performed over a broad temperature range $[80 \mathrm{~K}, 340 \mathrm{~K}]$. Pure tunneling gives rise to an ideality factor inversely proportional with temperature [2]. An ideality factor temperature law as $m(V, T) \sim T^{-\beta}$ with $0 \leq \beta \leq 1$ is a natural consequence of the Fermi-Dirac statistics as demonstrated above.

As a conclusion, we have shown that the ideality factor and barrier height essentially dependent on doping concentration and temperature. Moreover, the analysis demonstrates that the influence of the Fermi-Dirac statistics can cause effects previously attributed to tunneling only. Hence, both effects should be considered as suitable explanations for a nonBoltzmannian behavior of the ideality factor.

\section{APPENDIX}

\section{MATHEMATICAL DERIVATIONS}

\section{A. Derivation of (5)}

Combining (1) and (2) yields

$$
\frac{J_{n}(V)}{q \mu_{n}(x)}=\left(n(x ; V)-\frac{1}{f(x)} \frac{\partial n(x ; V)}{\partial \psi(x ; V)}\right) \frac{d \psi(x ; V)}{d x} .
$$

We use the identity

$$
\begin{aligned}
& -\frac{1}{f(x)} \exp \left(-\int_{x_{0}}^{x} \mathcal{E}(x ; V) f(x) d x\right) \frac{\partial}{\partial \psi(x ; V)} \\
& \times\left[n(x ; V) \exp \left(\int_{x_{0}}^{x} \mathcal{E}(x ; V) f(x) d x\right)\right] \\
= & \left(n(x ; V)-\frac{1}{f(x)} \frac{\partial n(x ; V)}{\partial \psi(x ; V)}\right)
\end{aligned}
$$

to obtain

$$
\begin{aligned}
& \frac{J_{n}(V)}{q D_{n}(x)} \exp \left(\int_{x_{0}}^{x} \mathcal{E}(x ; V) f(x) d x\right) \\
& =\frac{d}{d x}\left[n(x ; V) \exp \left(\int_{x_{0}}^{x} \mathcal{E}(x ; V) f(x) d x\right)\right] .
\end{aligned}
$$

We integrate from, say, $a$ to $b$ in the semiconductor side of the Schottky diode,

$$
\begin{aligned}
& \int_{a}^{b} \frac{J_{n}(V) d x}{q D_{n}(x)} \exp \left(\int_{x_{0}}^{x} \mathcal{E}(x ; V) f(x) d x\right) \\
& =n(b ; V) \exp \left(\int_{x_{0}}^{b} \mathcal{E}(x ; V) f(x) d x\right) \\
& -n(a ; V) \exp \left(\int_{x_{0}}^{a} \mathcal{E}(x ; V) f(x) d x\right) .
\end{aligned}
$$


Dividing both sides by $n(a ; V) \exp \left(\int_{x_{0}}^{a} \mathcal{E}(x ; V) f(x) d x\right)$ eliminates the arbitrary constant $x_{0}$,

$$
\begin{aligned}
& \frac{1}{n(a ; V)} \int_{a}^{b} \frac{J_{n}(V) d x}{q D_{n}(x)} \exp \left(\int_{a}^{x} \mathcal{E}(x ; V) f(x) d x\right) \\
& =\frac{n(b ; V)}{n(a ; V)} \exp \left(\int_{a}^{b} \mathcal{E}(x ; V) f(x) d x\right)-1
\end{aligned}
$$

The continuity equation for the electric current density, $\frac{d J_{n}(x ; V)}{d x}=0$ indicates that $J_{n}(x ; V)=J_{n}(V)$ is independent of position $x$. Rearrangement of (37) leads to (5). On the other hand, starting from the integral form (5), we easily recover (1) in the limit when $a$ tends to $b$. Indeed, application of theorem of de l'Hôpital and of (2) leads to (1). This demonstrates that (1) and (5) are mathematically equivalent.

\section{B. Estimates for the Ideality Factor $m(V, T)$}

We will present alternative expressions for the ideality factor

$$
\begin{aligned}
& \frac{1}{m(V, T)}=\frac{k_{B} T}{q V} \ln \left|\frac{J_{n}(V)}{J_{s}(V)}+1\right| \\
& =\frac{k_{B} T}{q V}\left(\ln \left|\frac{n(b ; V)}{n(a ; V)}\right|+\int_{a}^{b} \mathcal{E}(x ; V) f(x) d x\right) .
\end{aligned}
$$

Using the definition (2) of $f(x),(38)$ rewrites as

$$
\begin{aligned}
\frac{1}{m(V, T)}= & -\frac{k_{B} T}{q V}\left(\int_{a}^{b}\left[\frac{\partial \ln n(x ; V)}{\partial \psi(x ; V)}-\frac{\partial \ln n(x ; 0)}{\partial \psi(x ; 0)}\right]\right. \\
& \times \mathcal{E}(x ; V) d x) \\
= & \left.\frac{k_{B} T}{q V}\left(\int_{a}^{b} \frac{\partial \ln n(x ; v)}{\partial x} \frac{\mathcal{E}(x ; V)}{\mathcal{E}(x ; v)} d x\right)\right|_{v=0} ^{v=V} \\
= & \frac{k_{B} T}{q V}\left(\left.\ln n(x, v) \frac{\mathcal{E}(x ; V)}{\mathcal{E}(x ; v)}\right|_{a} ^{b}\right. \\
& \left.-\int_{a}^{b} \ln n(x ; v) \frac{\partial}{\partial x}\left(\frac{\mathcal{E}(x ; V)}{\mathcal{E}(x ; v)}\right) d x\right)\left.\right|_{v=0} ^{v=V} \\
= & \frac{k_{B} T}{q V}\left(\ln \left|\frac{n(b ; V)}{n(a ; V)}\right|-\left.\ln n(x, 0) \frac{\mathcal{E}(x ; V)}{\mathcal{E}(x ; 0)}\right|_{a} ^{b}\right. \\
& \left.+\int_{a}^{b} \ln n(x ; 0) \frac{\partial}{\partial x}\left(\frac{\mathcal{E}(x ; V)}{\mathcal{E}(x ; 0)}\right) d x\right)
\end{aligned}
$$

The point $b$ must be choosen in the neutral region where $\mathcal{E}(b ; V)=0$ for all voltages $V$. The last integral can be estimated in the assumption of the constant doping profiles and in the depletion approximation. We have that $\frac{\mathcal{E}(x ; V)}{\mathcal{E}(x ; 0)}=\frac{W_{d}(V)-x}{W_{d}(0)-x}=1-\frac{W_{d}(0)-W_{d}(V)}{W_{d}(0)-x}$ and $\frac{\partial}{\partial x}\left(\frac{\mathcal{E}(x ; V)}{\mathcal{E}(x ; 0)}\right)=$ $-\frac{W_{d}(0)-W_{d}(V)}{\left(W_{d}(0)-x\right)^{2}}<0$ in forward bias where $W_{d}(V)$ denotes the depletion thickness at applied voltage $V$. The last integral is thus negative. A simple estimate is

$$
\begin{aligned}
\int_{a}^{b} \ln n(x ; 0) \frac{\partial}{\partial x}\left(\frac{\mathcal{E}(x ; V)}{\mathcal{E}(x ; 0)}\right) d x> & \ln n(b ; 0) \int_{a}^{b} \frac{\partial}{\partial x} \\
& \times\left(\frac{\mathcal{E}(x ; V)}{\mathcal{E}(x ; 0)}\right) d x \\
= & -\ln n(b ; 0) \frac{\mathcal{E}(a ; V)}{\mathcal{E}(a ; 0)}
\end{aligned}
$$

such that, because $0<\frac{1}{m(V, T)} \leq 1$

$$
\begin{aligned}
\frac{1}{m(V, T)} \leq & \min \left\{\frac { k _ { B } T } { q V } \left(\ln \left|\frac{n(b ; V)}{n(a ; V)}\right|\right.\right. \\
& \left.\left.+\ln n(a, 0) \frac{\mathcal{E}(a ; V)}{\mathcal{E}(a ; 0)}\right), 1\right\} \\
> & \max \left\{\frac { k _ { B } T } { q V } \left(\ln \left|\frac{n(b ; V)}{n(a ; V)}\right|\right.\right. \\
& \left.\left.-\ln \left|\frac{n(b ; 0)}{n(a ; 0)}\right| \frac{\mathcal{E}(a ; V)}{\mathcal{E}(a ; 0)}\right), 0\right\} .
\end{aligned}
$$

\section{Short Note on the Fermi-Dirac Integrals $F_{p}(y)$}

A quite different manner to examine properties of Fermi-Dirac integrals consists in studying their generating function. When looking at the definition written as a $\wp-$ transform, where $\theta(x)$ is Heavyside's stepfunction,

$$
F_{p}(y)=\wp\left(\frac{x^{p} \theta(x)}{\Gamma(p+1)}, y\right)=\frac{1}{\Gamma(p+1)} \int_{0}^{\infty} \frac{x^{p} d x}{1+\exp (x-y)}
$$

we may interprete $\Gamma(p+1) F_{p}(y)$ as the Mellin transform of the function $(1+\exp (x-y))^{-1}$, which alternatively means by inversion,

$$
\frac{1}{1+\exp (x-y)}=\frac{1}{2 \pi i} \int_{k-i \infty}^{k+i \infty} \Gamma(p) F_{p-1}(y) x^{-p} d p \quad(k>0) \text {. }
$$

We now need the following important property of the Mellin transform. If $F(s)$ and $G(s)$ are the Mellin transform of the absolute integrable functions on $[0, \infty), f(x)$ and resp. $g(x)$, and if $k$ and $\alpha$ are real numbers suitably chosen in order that $x^{k} f(x)$ and $x^{\alpha-k} g(x)$ both belong to $\mathcal{L}^{2}$, the class of absolute integrable functions on $[0, \infty]$, then holds [22]

$$
\int_{0}^{\infty} f(x) g(x) x^{s-1} d x=\frac{1}{2 \pi i} \int_{k-i \infty}^{k+i \infty} F(w) G(s-w) d w .
$$

The interest of relation (45) is that using (45) the single sided $\wp-$-transform can be written in terms of the Fermi-Dirac integrals provided that the Mellin transform $G(s)$ of the density function $g(x)$ exists. $^{2}$

$$
\begin{aligned}
n(y) & =\wp(g(x) \theta(x), y)=\int_{0}^{\infty} \frac{g(x)}{1+\exp (x-y)} d x \\
& =\frac{1}{2 \pi i} \int_{k-i \infty}^{k+i \infty} \Gamma(1-s) F_{-s}(y) G(s) d s .
\end{aligned}
$$

\footnotetext{
${ }^{2}$ It is very likely that there exists a nonzero s-interval around $s=1$ where
} $G(s)$ converges since for density functions $\int_{0}^{\infty} x^{s-1} g(x) d x=1$ for $s=1$. 
This demonstrates that we may rewrite the number of electron $n(y)$ for an arbitrary density of states in terms of the Fermi-Dirac integrals.

\section{ACKNOWLEDGMENT}

The author is much indebted to Professor J. A. del Alamo of MIT.

\section{REFERENCES}

[1] S. S. Cohen and G. Sh. Gildenblat, "VLSI electronics microstructure science," in Metal-Semiconductor Contacts and Devices, vol. 13. New York: Academic, 1986.

2] F A Padovani, "The voltage-current characteristic of metalsemiconductor contacts," in Semiconductors and Semimetals, R. K Willardson and A. C. Beer, Eds. New York: Academic, 1971, vol. 7a, pp. $75-146$.

[3] R. J. Van Overstraeten, H. J. De Man, and R. P. Mertens, "Transport equations in heavily doped silicon," IEEE Trans. Electron Devices, vol. ED-20, pp. 290-298, Mar. 1973.

[4] R. P. Mertens, R. J. Van Overstraeten, and H. J. De Man, Advances in Electronics and Electron Physics, vol. 55. New York: Academic, 1981 , p. 77.

[5] W. Hänsch, "The drift diffusion equation and its applications in MOS FET modeling," in Computational Micro-Electronics, S. Selberherr, Ed Wien: Springer-Verlag, 1991.

[6] P. Van Mieghem, R. P. Mertens, and R. J. Van Overstraeten, "Theory of the junction capacitance of an abrupt diode," J. Appl. Phys., vol. 67, no. 9, pp. 4203-4211, 1990

[7] P. Van Mieghem, "Origin of the difference between the capacitance intercept voltage and the built-in potential", IEEE Trans. Electron Devices, vol. 40, pp. 2365-2368, Dec. 1993.

[8] P. Van Mieghem and R. Mertens, "Heavy doping effects in silicon," chap. 1 in Selected Topics on the Physics and Technology of Advanced Semiconductor Silicon- and Silicon Alloy-Based Devices, J. Nijs, Ed. Brussels: Adam Hilger, 1994.

[9] P. Van Mieghem, "Theory of bandtails in heavily doped semiconductors," Rev. Mod. Phys., vol. 64, no. 3, pp. 755-794, 1992.

[10] R. B. Dingle, "The Fermi-Dirac integrals," Appl. Sci. Res. B, vol. 6, pp. $225-239,1956$

111] C. R. Crowell and S. M. Sze, "Current transport in metal-semiconductor barriers," Solid-State Electron., vol. 9, pp. 1035-1048, 1966.
[12] J. G. Simmons and G. W. Taylor, "Generalized theory of conduction in Schottky barriers," Solid-State Electron., vol. 26, pp. 705-709, 1983.

[13] B. Vinter. "Influence of charged impurities on Si inversion-layer.electrons," Phys. Rev. B, vol. 26, no. 12, pp. 6808-6825, 1982.

[14] S. M. Sze, Physics of Semiconductor Devices. New York: Wiley, 1981

[15] P. M. Morse and H. Feshbach, Methods of Theoretical Physics. New York: McGraw-Hill, 1978

[16] L. D. Landau and E. M. Lifshitz, Statistical Physics. Oxford, UK: Pergamon, 1980.

[17] J. S. Blakemore, Semiconductor Statistics. New York: Pergamon, 1962, Appendix C.

[18] R. T. Tung, J. P. Sullivan, D. J. Eaglesham, and F. Schrey, Schottky Barrier Heights of Epitaxial Sillicides, Electron. Material Conf., Santa Barbara, CA, Abstracts A2 (invited), June 1993.

[19] R. T. Tung, "Electron transport at the metal-semiconductor interfaces: General theory," Phys. Rev. B vol. 45, no. 23, pp. 13509-13523, 1992.

[20] J. P. Sullivan, R. T. Tung, M. R. Pinto, and W. R. Graham, "Electron transport of inhomogeneous Schottky barriers: A numerical study," $J$. Appl. Phys., vol. 70, no. 12, pp. 7403-7424, 1991.

[21] C. J. Palmstrøm and D. V. Morgan, "Metallizations for GaAs devices and circuits," in Gallium Arsenide, M. J. Howes and D. V. Morgan, Eds. Wiley, pp. 195-261, 1985.

[22] E. C. Titchmarsh, Introduction to the Theory of Fourier Integrals. Oxford, UK: Oxford Univ. Press, 1948

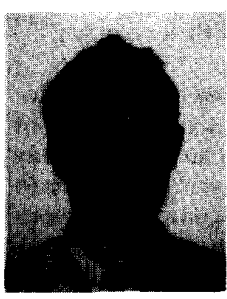

Piet Van Mieghem was born in Ninove, Belgium, in 1964. He received the M.S. degree in electrical engineering from the Katholieke Universiteit Leuven (KUL) in 1987. In 1991 he obtained the Ph.D. in electrical engineering from KUL for his dissertation on heavy doping effects in semiconductors.

In 1987, he joined the Interuniversity Micro Electronics Center, Leuven. During 1993, he was a Postdoctoral Scientist at the Massachusetts Institute of Technology (MIT). Currently he is working in the corporate research center of Alcatel-Bell at Antwerpen where he is engaged in call acceptance and congestion control of ATM networks.

Dr. Van Mieghem was awarded the Student Prize in 1987 for the best Belgian Master's thesis in electrical engineering by the Belgian Institute for Control and Automatization (BIRA). 